\title{
Modeling of Needle-Tissue Interaction Using Ultrasound-Based Motion Estimation
}

\author{
Ehsan Dehghan ${ }^{1}, \mathrm{Xu}$ Wen $^{1}$, Reza Zahiri-Azar ${ }^{1}$, Maud Marchal $^{1,2}$, \\ and Septimiu E. Salcudean ${ }^{1}$ \\ ${ }^{1}$ Department of Electrical and Computer Engineering, \\ University of British Columbia, Vancouver, Canada \\ tims@ece.ubc.ca \\ 2 TIMC-GMCAO Laboratory, Grenoble, France
}

\begin{abstract}
A needle-tissue interaction model is an essential part of every needle insertion simulator. In this paper, a new experimental method for the modeling of needle-tissue interaction is presented. The method consists of measuring needle and tissue displacements with ultrasound, measuring needle base forces, and using a deformation simulation model to identify the parameters of a needle-tissue interaction model. The feasibility of this non-invasive approach was demonstrated in an experiment in which a brachytherapy needle was inserted into a prostate phantom. Ultrasound radio-frequency data and the time-domain cross-correlation method, often used in ultrasound elastography, were used to generate the tissue displacement field during needle insertion. A three-parameter force density model was assumed for the needle-tissue interaction. With the needle displacement, tissue displacement and needle base forces as input data, finite element simulations were carried out to adjust the model parameters to achieve a good fit between simulated and measured data.
\end{abstract}

\section{Introduction}

During prostate brachytherapy, radioactive capsules are implanted inside the prostate and the surrounding tissue using a long needle with visual guidance from trans-rectal ultrasound (TRUS) and real-time X-ray fluoroscopy. The success of brachytherapy relies on the accuracy of the needle placement inside the tissue. However, due to prostate deformation and rotation [1], targeting errors are still common in brachytherapy [2] and can result in under-dosed and over-dosed regions that can lead to repeated treatments or complications, such as impotence or urinary incontinence. Since visual feedback is limited, significant skill is required to compensate for tissue deformation and decrease targeting errors. Brachytherapy simulators 34 and path planners represent new alternatives to train physicians and provide pre-operative planning.

There has been extensive research to model the needle-tissue interactions during needle insertion [5,6/7,8,9,10]. Okamura et al. [5] inserted a needle into bovine liver and divided the force applied by the tissue to the needle into three parts: 1) capsule stiffness; 2) friction and 3) cutting forces occurring at the needle

N. Ayache, S. Ourselin, A. Maeder (Eds.): MICCAI 2007, Part I, LNCS 4791, pp. 709-716, 2007.

(C) Springer-Verlag Berlin Heidelberg 2007 
tip. The authors did not track tissue displacements in their work. Kataoka et al. [6] reported the tip and friction forces applied by a needle during penetration into a canine prostate. Podder et al. 7] reported the needle forces measured during brachytherapy of 25 patients and developed a patient-specific and procedurespecific statistical model to estimate the maximum force that the needle will experience during insertion into the prostate and the perineum. DiMaio and Salcudean 8 identified the force profile along the needle during penetration into a slab of PVC by tracking the motion of superficial markers using a camera. They identified a force model with a peak at the needle tip, following a constant shaft force density. Hing et al. 9] tracked the displacements of several implanted fiducial beads during needle insertion using a dual C-arm fluoroscope setup. They identified a local effective modulus during puncture and an approximate cutting force for soft tissue samples. Crouch et al. [10] introduced a velocity-dependent needle shaft force density. This model has a constant shaft force density followed by a dip and a peak at the tip. The force-displacement data were acquired from insertion of a needle into a transparent, homogeneous silicone gel phantom in which several layers of fiducial markers were implanted. Two digital cameras were used to track the movement of fiducial markers in 3-D.

In this paper, a new experimental method is proposed in order to model needle insertion into soft tissues. The method consists of measuring tissue displacements with ultrasound radio-frequency (RF) data, measuring needle base forces, and using a deformation simulation model to identify the parameters of a needle-tissue interaction model. The use of ultrasound imaging for tissue deformation measurement has several advantages: it is non-invasive and safe, it is the main imaging modality during many image-guided procedures such as prostate brachytherapy, and it does not require fiducial markers. The feasibility of this non-invasive approach was demonstrated in an experiment in which a brachytherapy needle was inserted into a non-homogeneous phantom composed of a harder inclusion mimicking the prostate and a softer surrounding tissue. The Time-Domain Cross-Correlation with Prior Estimates (TDPE) 11,12 was used to estimate the tissue displacements from ultrasound RF signals. This method has the ability to estimate the displacements in real-time. The RF correlation approach has demonstrated high resolution in elastography, hence high accuracy can be expected.

The paper is divided into five sections. In Sect. 2, the experiment design is detailed. Section 3 presents the measurements obtained. Section 4 proposes the modeling method. Section 5 draws conclusions and discusses future work.

\section{Experiment Setup}

An experiment was conducted to measure both the forces applied on a needle during its insertion into soft tissue and the resulting tissue displacements. The apparatus consists of a needle insertion device, allowing controlled insertion of a needle into a phantom, and an ultrasound machine used to track the tissue displacements. 


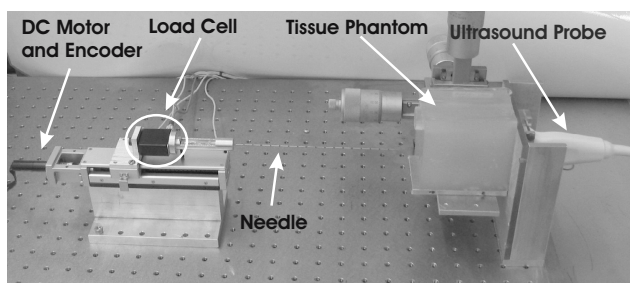

(a)

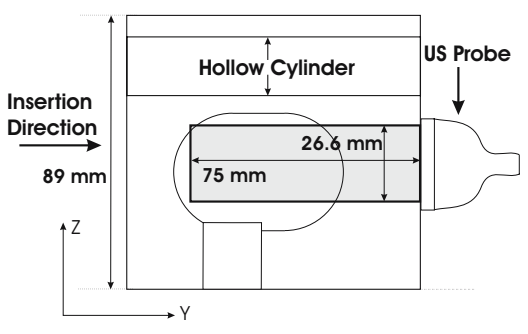

(b)

Fig. 1. (a) The experiment setup and (b) Side view of the phantom, showing the inclusion, hollow cylinder and the US field of view

\subsection{Needle Insertion Device}

An 18 gauge brachytherapy needle (Bard, NJ, USA) was mounted on a translational lead-screw stage powered by a Maxon DC motor with an optical encoder. A proportional controller was used to control the speed of the heavily geared drive motor. The needle was mounted on a load cell (MBD-2.5 Transducer Techniques, CA, USA) to measure the insertion and retraction forces applied on it. A computer was used to control the needle speed and to record the needle position and the feedback force at $20 \mathrm{~Hz}$. The experiment setup is shown in Fig. 1(a).

\subsection{Phantom Construction}

A non-homogeneous phantom composed of a harder inclusion surrounded by a softer tissue has been constructed for the experiments. Fig.1(b) shows its schematic diagram. The harder inclusion of the phantom - designed to mimic the prostate - is a cylinder with two hemispheres at the two ends. This inclusion was made from polyvinyl chloride (PVC) plasticizer (M-F Manufacturing Co., Inc. Fort Worth, TX, USA). The outside substrate was made from $66.7 \%$ PVC plasticizer and 33.3\% plastic softener (M-F Manufacturing Co., Inc. Fort Worth). The inclusion was connected to the base with a cylinder of the same material to mimic the rotation of the prostate around the pubic bone. Cellulose (SigmaAldrich Inc., St. Louis, MO, USA) was added to the two parts as scattering particles. A cylindrical hole through the phantom represents the rectum. A stiff cylinder made of hard plastic was inserted into this hole to simulate the rectal probe and its effects on the motion of the prostate.

\subsection{Tissue Deformation Tracking}

A Sonix RP PC-based ultrasound machine and an L12-5 38-mm linear probe (Ultrasonix Medical Corp., Burnaby, BC, Canada) were used in the experiments. 


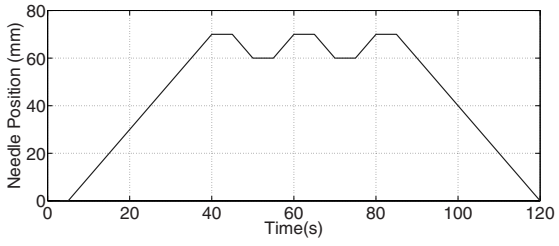

(a)

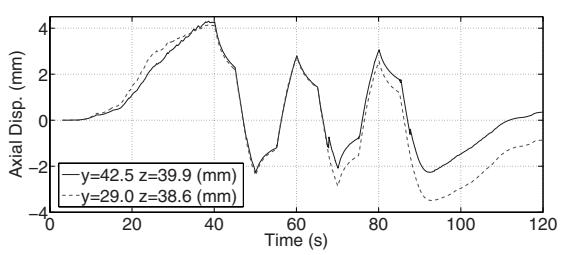

(c)

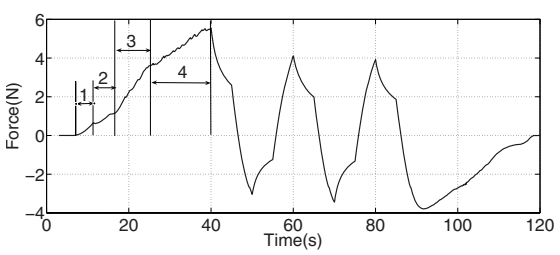

(b)

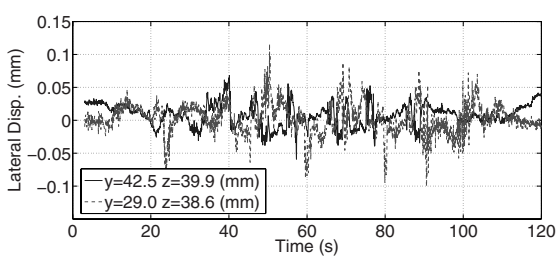

(d)

Fig. 2. (a) Needle tip position, (b) Measured insertion force, (c) Axial and (d) Lateral displacements of two sample nodes located in the ultrasound field of view. The legends show the initial location of the nodes. The needle was partially retracted and inserted again after the main insertion. Since in the second and third insertions the needle was inserted in the same path as the first insertion, no cutting occurred. Therefore, the second and third peak forces $(t=60$ and $80 \mathrm{~s})$ are smaller than the first one $(t=40 \mathrm{~s})$.

Both B-mode ultrasound images and digitized radio-frequency $(\mathrm{RF})$ signals were acquired simultaneously with this machine. The machine was synchronized with the computer, which controlled the insertion device and recorded the force data. The phantom was imaged to a depth of $75 \mathrm{~mm}$ using a linear array of $128 \mathrm{el}-$ ements with 1.6 lines per millimeter in the lateral direction ( $70 \%$ sector). The centroid frequency was $5 \mathrm{MHz}$. RF frames were captured in real-time at 20 frames per second. The position of the ultrasound probe with respect to the tissue and the US field of view inside the tissue are shown in Fig.1(b).

TDPE [11]12 was used to process the data off-line. Each RF-line was divided into 120 overlapping windows ( $1 \mathrm{~mm}$ window length and $60 \%$ window overlap). The axial [1] and lateral [12] components of the displacement (along $y$ and $z$ axes as shown in Fig.1(b) were estimated from RF frames. In this method, absolute motions are estimated by integration of relative motions. To increase the accuracy of the estimation, the following dynamic reference frame updating algorithm was used. For every RF frame, the displacements were estimated with respect to the reference frame which was originally set as the first frame. To compensate for RF de-correlation resulting from large displacements, the reference frame was moved to the latest estimated displacement, as soon as the average correlation coefficient corresponding to the latest simulated displacement dropped below 0.95. At each step, the absolute displacements for every spatial location were reported as the estimated motions at that location added to the accumulated displacement value in the integrator. 


\section{Force and Displacement Measurements}

The needle was inserted along the $y$ axis with a controlled position as shown in Fig.2(a) The insertion line was $5 \mathrm{~mm}$ out of the ultrasound field of view to avoid the deteriorating effects of a metallic object on the US images and to increase the accuracy of the tracking algorithm. The tissue phantom was meshed using tetrahedral elements to be used in a model based on the finite element method (FEM) as described in Sect. 4. Some of the mesh nodes were located in the ultrasound field of view. The axial and lateral displacements of these nodes were measured during the experiment (see Figs.2(c) and 2(d)). Due to the higher accuracy and resolution in the axial direction, only axial displacement estimations were used for modeling. The needle was fully retracted at $t=118.5 \mathrm{~s}$, since the measured force is zero after this time (see Fig.2(b)]. However, the measured nodal displacements in Fig.2(c) show non-zero displacements after this time, which is due to the accumulation of residuals caused by integration of relative motions and the topological change caused by the needle insertion. The characterization of this drift and its effect on the identified model is the subject of future research. The measured force is shown in Fig.2(b). The decrease in force noted when the needle stops moving is due to tissue relaxation.

\section{Needle Shaft Force Distribution}

A force distribution as shown in Fig. 3(b) was adopted to model the needle-tissue interaction. This force distribution has three parameters, a constant shaft force density $f_{\mathrm{s}}$ that can simulate friction, a peak force density $f_{\mathrm{p}}$ over the area close to the tip, which contributes to the cutting force, and the width of the peak force density, $w$. This choice was inspired by the measured force in Fig.2(b) which shows four parts during insertion into two different tissue types and by the model presented in $[8]$. To identify the parameter values of this model, only the data from the first insertion portion $(0 \leq t \leq 40 \mathrm{~s})$ are used. Therefore, the tissue relaxation is not considered in the modeling part. In addition, the overall drift error is less than $1 \mathrm{~mm}$ over 120 seconds, while the maximum displacement, of the order of $4 \mathrm{~mm}$, takes place in the first 40 seconds of the experiment. Therefore, the drift error is assumed to be negligible during the first insertion portion.

The tissue phantom was meshed using 991 nodes and 4453 linear tetrahedral elements to be used in a finite element analysis. The needle insertion process was simulated using the FEM. Since the tissue is confined in this experiment and is not allowed to rotate easily, a linear FEM model can be used as opposed to a non-linear one [13. Due to the slow speed of insertion, the velocity dependent properties of tissue were neglected and the simulation was performed in a quasi-static mode. The force profile shown in Fig. 3(b) was implemented in the simulator and the corresponding displacements for nodes in the US field of view were simulated. In the simulation program, the shaft force density was integrated 


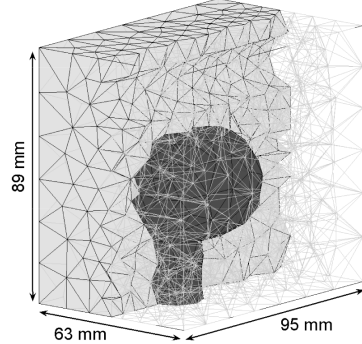

(a)

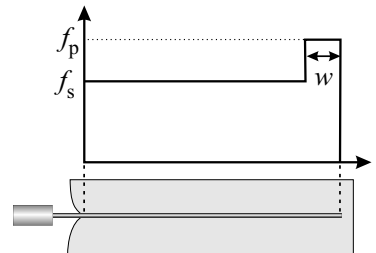

(b)

Fig. 3. (a) Tissue phantom meshed with tetrahedral elements and (b) The needle shaft force distribution

Table 1. Needle shaft force distribution and elastic parameters

\begin{tabular}{lcccc}
\hline & $f_{\mathrm{s}}(\mathrm{N} / \mathrm{m})$ & $f_{\mathrm{p}}(\mathrm{N} / \mathrm{m})$ & $w(\mathrm{~mm})$ & Young's Modulus $(\mathrm{kPa})$ \\
\hline Inclusion & 72 & 320 & 7.0 & 10 \\
Surrounding tissue & 60 & 140 & 4.0 & 7 \\
\hline
\end{tabular}

over the part of the needle which was inside the deformed tissue. This force was distributed over the nodes in contact with the needle. The nodes located on the bottom and back surfaces of the phantom and the nodes in contact with the stiff cylinder were fixed. The force distribution parameters were adjusted to fit the simulated force to the measured force. Since the tissue elastic parameters were unknown prior to the experiment, they were adjusted in the simulation program to fit the simulated axial displacements to the measured axial displacements. The Poisson's ratio was assumed to be equal to 0.49 to simulate the near incompressibility of tissue. The tissue force distribution and material elastic parameters are shown in Table1 for the given phantom.

The simulated and measured forces are shown in Fig.4(a). This figure shows the ability of the proposed force distribution model to simulate the needle force with high accuracy. The maximum error between simulated and measured forces is $0.33 \mathrm{~N}$. The minor discontinuity in the simulated force around $t=20 \mathrm{~s}$ is caused by the node repositioning method [4] used in the simulation program to increase the accuracy. Figure 4(b) shows the average simulated and measured axial displacements for the nodes in the ultrasound field of view. The simulated axial displacement has a maximum average error of $0.1 \mathrm{~mm}$ and standard deviation of $1.2 \mathrm{~mm}$. Fig. 4(c) shows the position of the nodes in the US field of view in the deformed and undeformed configurations.

In the work presented, the elastic and force model parameters of two tissue types were identified. However, if the elastic parameters are identified using other methods prior to the modeling, the force model parameters can be identified for several layers of tissue. If the elastic parameters are unknown, the identification process for several tissue layers will be complicated, due to mutual effects of one layer of tissue on the displacement of the other ones. 


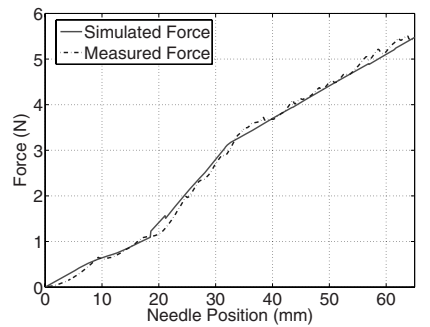

(a)

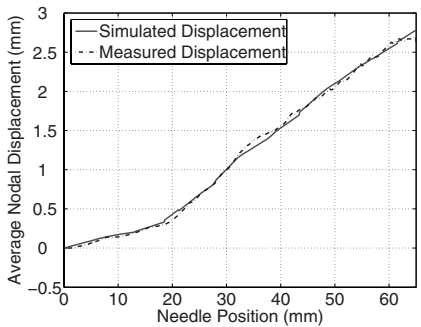

(b)

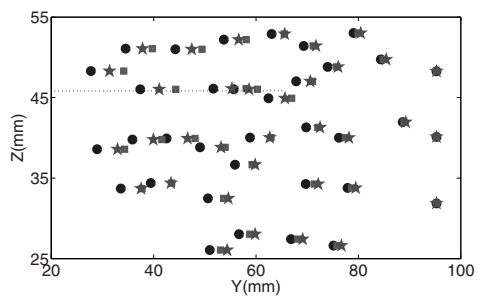

(c)

Fig. 4. (a) Simulated and measured insertion forces, (b) Simulated and measured average of nodal displacements in the axial direction, and (c) Position of the nodes in the US field of view. Only axial displacements are shown and were considered in identifying the model parameters. Circles denote the original positions, squares simulated positions and stars the positions measured with TDPE. The dotted line shows the projection of the needle on the US plane.

\section{Conclusion and Future Work}

A new experimental method has been presented to study and model the needletissue interactions. The method is based on measuring the tissue displacement from ultrasound RF data and measuring needle forces and needle base position during the insertion process. A three parameter force distribution model for the needle-tissue interactions has been presented. The model parameters were adjusted using the measurements of force-displacement data recorded during insertion of a needle into a non-homogeneous PVC phantom. The tissue phantom was composed of a harder inclusion to simulate the prostate and a softer surrounding tissue. It was not transparent and had no fiducial beads implanted in. An FEM based simulation was used to adjust the parameters and fit the simulated and measured forces. In addition, the Young's moduli of the tissues were adjusted to fit the simulated axial displacements to the measured axial displacements. The identified force profile and the elastic properties can be used to construct an FEM simulator to simulate the needle insertion process. Such a simulator can be helpful for path planning and for physician training.

In the future, the elastic parameters of the tissue will be identified with higher accuracy using a finer mesh. The dependency of the force model parameters to the insertion speed and the viscoelastic properties of the tissue will be 
investigated. Statistical analysis will be carried out by acquiring more insertion data from the phantom. In addition, the tracking algorithm will be validated by implanting beads in the tissue and by comparing TDPE results with other imaging modalities and tracking algorithms. During brachytherapy, images are acquired using TRUS. Therefore, the needle motion and major tissue displacements are in the lateral direction of the sagittal/parasagittal images. However, lateral displacement estimation has resolution that is one order of magnitude lower than the resolution in the axial direction. The effect of this discrepancy of resolution on the model depends on the tissue homogeneity and isotropy and will be the subject of further research.

\section{References}

1. Lagerburg, V., Moerland, M.A., Lagendijk, J.J., Battermann, J.J.: Measurement of prostate rotation during insertion of needles for brachytherapy. Radiotherapy and Oncology 77, 318-323 (2005)

2. Teschereau, R., Pouliot, J., Roy, J., Tremblay, D.: Seed misplacement and stabilizing needles in transperineal permanent prostate implants. Radiotherapy and Oncology 55, 59-63 (2000)

3. Alterovitz, R., Pouliot, J., Taschereau, R., Hsu, I.C., Goldberg, K.: Needle insertion and radioactive seed implantation in human tissue: Simulation and sensitivity analysis. In: Proc. IEEE ICRA pp. 1793-1799 (2003)

4. Goksel, O., Salcudean, S.E., DiMaio, S.P.: 3D simulation of needle-tissue interaction with application to prostate brachytherapy. Computer Aided Surgery 11(6), 279-288 (2006)

5. Okamura, A., Simone, C., O'Leary, M.: Force modeling for needle insertion into soft tissue. IEEE Trans. Biomed. Eng. 51, 1707-1716 (2004)

6. Kataoka, H., Washio, T., Chinzei, K., Mizuhara, K., Simone, C., Okamura, A.: Measurement of tip and friction force acting on a needle during penetration. In: Dohi, T., Kikinis, R. (eds.) MICCAI 2002. LNCS, vol. 2488, pp. 216-223. Springer, Heidelberg (2002)

7. Podder, T., Sherman, J., Messing, E., Rubens, D., Fuller, D., Strang, J., Brasacchio, R., Yu, Y.: Needle insertion force estimation model using procedure-specific and patient-specific criteria. In: Proc. IEEE EMBS Int. Conf, pp. 555-558 (2006)

8. DiMaio, S.P., Salcudean, S.E.: Needle insertion modeling and simulation. IEEE Trans. on Robot. Autom.: Special Issue on Medical Robotics 19, 864-875 (2003)

9. Hing, J.T., Brooks, A.D., Desai, J.P.: Reality-based needle insertion simulation for haptic feedback in prostate brachytherapy. In: Proc. IEEE ICRA, pp. 619-624 (2006)

10. Crouch, J.R., Schneider, C.M., Wainer, J., Okamura, A.M.: A velocity-dependent model for needle insertion in soft tissue. In: Duncan, J.S., Gerig, G. (eds.) MICCAI 2005. LNCS, vol. 3750, pp. 624-632. Springer, Heidelberg (2005)

11. Zahiri-Azar, R., Salcudean, S.E.: Motion estimation in ultrasound images using time domain cross correlation with prior estimates. IEEE Trans. Biomed. Eng. 53(10), 1990-2000 (2006)

12. Zahiri-Azar, R., Salcudean, S.E.: Real-time estimation of lateral motion using time domain cross correlation with prior estimates. In: Proc. IEEE Ultrasonics Symposium, pp. 1209-1212 (2006)

13. Dehghan, E., Salcudean, S.E.: Comparison of linear and non-linear models in 2D needle insertion simulation. In: Proc. Workshop on Computational Biomechanics for Medicine (In conjunction with MICCAI 2006), pp. 117-124 (2006) 